\title{
Sociedad de plataformas: economía y regulación
}

Platform Society: Economy and Regulation

Societat de plataformes: economia i regulació

Rayner García Hernández iD

Investigador independiente

tangaray83@gmail.com

Recibido: 10/09/2021

Aceptado: $14 / 12 / 2021$ 
Resumen Tomando en consideración que los medios digitales se constituyen como aspectos culturales esenciales de la realidad social actual, el artículo propone un acercamiento al término sociedad de plataformas para analizar la relación compleja entre las acciones de los consorcios de Internet, las rutinas de uso para un manejo productivo de la tecnología digital y el creciente papel de la tecnología en la vida cotidiana. Desde un enfoque sociológico y praxeológico, se describen algunos fenómenos que caracterizan y describen la economía de las plataformas digitales y el proceso de regulación de su infraestructura. En las plataformas digitales se utiliza un amplio espectro de prácticas mediáticas que penetran profundamente en los entornos sociales a partir de las normas y políticas que establecen. Éstas se utilizan como mecanismos reguladores de la actividad y el comportamiento de los usuarios en la infoesfera, en tanto los consorcios de Internet desvían, analizan y procesan grandes cantidades de datos y los clasifican como materias primas. Así, se plantea la tesis que las plataformas digitales con sus infraestructuras técnicas orientadas a la prestación de servicios, generan una cultura organizacional que transforma los entornos sociales y modifica las prácticas mediáticas.

Palabras Sociedad de plataformas, economía, regulación, prácticas, entornos sociales.

clave

\begin{abstract}
Taking into consideration that digital media are constituted as essential cultural aspects of current social reality, the article proposes an approach to the term platform society to investigate the complex relation between the actions of Internet consortia, the routines of use towards a productive management of digital media and the growing role of technology in everyday life. From a sociological and praxeological point of view, this article characterizes and describes some phenomena of the digital platforms economy and the process of regulating their infrastructure. In the digital platforms is used a wide spectrum of media practices that penetrate deeply into social settings. These practices are based on the norms and policies that the platforms establish. Thus, these are also used in the infosphere as regulatory mechanisms of the users activity and behavior, while Internet consortia divert, analyze and process large amounts of data and classify them as raw materials. The goal of this article is to examine the thesis, that digital platforms with their technical infrastructures oriented to services provision, generate an organizational culture that transforms social settings and modifies media practices.
\end{abstract}

Keywords Platform Society, Economy, Regulation, Practices, Social Settings.

Resum

Paraules

clau
Prenent en consideració que els mitjans digitals es constitueixen com a aspectes culturals essencials de la realitat social actual, l'article proposa un acostament al terme societat de plataformes per a analitzar la relació complexa entre les accions dels consorcis d'Internet, les rutines d'ús per a un maneig productiu de la tecnologia digital i el creixent paper de la tecnologia en la vida quotidiana. Des d'un enfocament sociològic i praxiològic, es descriuen alguns fenòmens que caracteritzen i descriuen l'economia de les plataformes digitals i el procés de regulació de la seva infraestructura. En les plataformes digitals s'utilitza un ampli espectre de pràctiques mediàtiques que penetren profundament en els entorns socials a partir de les normes i polítiques que estableixen. Aquestes s'utilitzen com a mecanismes reguladors de l'activitat i el comportament dels usuaris en la infoesfera, en tant els consorcis d'Internet desvien, analitzen i processen grans quantitats de dades i les classifiquen com a matèries primeres. Així, es planteja la tesi que les plataformes digitals amb les seves infraestructures tècniques orientades a la prestació de serveis, generen una cultura organitzacional que transforma els entorns socials i modifica les pràctiques mediàtiques.

Societat de plataformes, economia, regulació, pràctiques, entorns socials. 


\section{Introducción}

A partir del supuesto de que los contextos sociales contemporáneos están saturados con procesos técnicos de mediación y digitalización, el presente artículo se apoya en la idea mcluhaniana de los medios como environment - es decir entornos -, para analizar algunos de los efectos de la tecnología y la comunicación digital en la actividad de los individuos. El enfoque sociológico y praxeológico (Schäfer, 2016, Schatzki, 2016, Reckwitz, 2003) del presente artículo se orienta a indagar en las prácticas mediáticas de los consorcios de Internet, además de analizar algunos aspectos del uso cotidiano de la tecnología digital como elemento esencial no separable de la praxis social. Las tecnologías digitales se integran en la vida cotidiana y se adaptan a las prácticas diarias (Silverstone y Hirsch, 1992), en tanto los usuarios y su entorno se transforman y se adaptan en correspondencia con las transformaciones que las plataformas digitales producen.

El foco de la investigación recae en las acciones de las plataformas digitales, las prácticas de los usuarios y el entorno mediático de su implementación en un contexto transcultural, con el objetivo de reflexionar no solo sobre la adaptación, recepción y uso de formatos digitales, sino también sobre la influencia de la tecnología en la conformación de entornos sociales. En sentido transcultural, Arjun Appadurai (1996) recomienda el uso de la palabra culturalismo para denotar la movilización social y el desarrollo de procesos políticos de formación de identidad en los que los medios de comunicación y la fuerza imaginativa - entendida como habilidad creativa del ser humano - desempeñan un rol cada vez más relevante en nuestras sociedades contemporáneas. La comprensión del espacio - digital y analógico - en el que las plataformas digitales y los usuarios ejercen sus prácticas debe entenderse como un lugar de observación y orientación humanas. Éste no puede describirse sin tener en cuenta la fuerza constitutiva de las diversas técnicas culturales, los medios de comunicación y los procesos económicos, políticos y sociales que lo conforman (Günzel, 2010, p. XI).

El contexto en el cual se desarrolla la denominada sociedad de plataformas (van Dijck et al., 2018, Nash et al., 2017) está caracterizado por el diseño liberal y supuestamente emancipador de los dispositivos técnicos, visiones neoliberales de mercado y entornos digitales deterministas en donde las redes sociales ocupan un lugar central. Mediante la creciente digitalización y mediatización de la vida cotidiana, así como a través de sus estrategias de innovación, los consorcios de Internet no sólo diseñan y crean ofertas, infraestructuras y modelos de mercado que organizan, estructuran y manipulan las opciones de comunicación de los usuarios, sino también regulan el acceso a la red y centralizan el poder ejerciendo prácticas de control en las plataformas digitales. De esta manera, estas plataformas se erigen como modelos de negocio en las que mediante su uso, cada aspecto de la vida social es propenso a 
ser expuesto, dilucidado y gestionado (Fernández, 2018). Considerando algunas de las formas de comunicación, así como algunas de las prácticas mediáticas, el artículo se acerca además a los términos economía de plataformas (Busch 2021, Dolata, 2020, Féliz y Bona 2020) y capitalismo digital (Staab, 2019, Srnicek, 2018, Schiller, 2014) para indagar en los procesos actuales de regulación jurídica de Internet.

Para alcanzar los objetivos, se plantea la tesis que las plataformas digitales con sus infraestructuras técnicas orientadas a la prestación de servicios, generan una cultura organizacional que transforma los entornos sociales y modifica las prácticas mediáticas. Para ello, se propone la hipótesis de que desde finales de los años 1990 e inicios de los 2000, el Internet experimenta un cambio tecnológico gradual de su dimensión cultural a una netamente comercial, donde es perceptible una transición del paradigma de la industria a uno relacionado, principalmente, con la prestación de servicios. Manuel Castells (2002) expresaba que esta dimensión cultural se constituye en y desde el sistema de valores, creencias y formas de construir mentalmente una sociedad, la cual es decisiva en la producción de los formatos que conforman el Internet.

\section{Entornos sociales y prácticas mediáticas}

Desde el diseño de una socialidad técnicamente expandida (Dolata y Schrape, 2018, Stalder, 2016), los usuarios intervienen cada vez más en nuevos terrenos de la infoesfera (Floridi, 2015). Mediante el uso de tecnologías digitales complejas, pero fáciles de manejar, ellos participan en la negociación de nuevos significados culturales, políticos y sociales (García Canclini, 2020). Con la ayuda de los diversos formatos digitales para la comunicación, los usuarios reaccionan a los efectos y desafíos de un espacio informativo novedoso - dígase Internet y sus servicios - y contribuyen a su difusión con sus acciones en el entorno digital, por ejemplo, mediante el uso del Smartphone.

Desde la teoría de domesticación (Silverstone y Hirsch, 1992) se enfatiza el rol de la subjetividad de los usuarios frente a la posibilidad de producir, diseñar y transformar objetos, significados y valoraciones culturales precisamente a través de su consumo, apropiación de contenidos y el uso cotidiano de los dispositivos técnicos. Este sensorium (Martín Barbero, 2010) que encarnan los usuarios por medio del uso de la tecnología se refiere a esa percepción, apropiación e interpretación de la información que utilizan y la influencia de ésta en sus experiencias en el entorno digital. La forma en cómo se producen y construyen las infraestructuras técnicas para el consumo de contenidos digitales, además de la manera en cómo se traduce su uso en los diversos contextos, generan realidades alternas con significados compartidos por una gran cantidad de usuarios. Para Jesús Martín Barbero (2010) se hace necesario comprender esta densidad de las transformaciones de los modos de 
comunicar como la aparición de una experiencia cultural nueva, pues así se modifican, en su esencia, las maneras de percibir y sentir, de escuchar y de ver. Las transformaciones constantes en los procesos de innovación de los consorcios de Internet constituyen elementos fundamentales para el desarrollo de una sensibilidad colectiva cambiante.

El grupo denominado como los GAFA (Google, Amazon, Facebook y Apple) crea el marco jurídico o legal para generar contextos sociales digitales, donde los usuarios establecen redes de relaciones a partir de su interacción con la tecnología y desde perfiles individualizados orientados al marketing y la publicidad. En el mercado digital actual, los usuarios interactúan con productos y mercancías que tienen como objetivo regular la oferta y la demanda. En los dispositivos digitales y, sobre todo, mediante el uso de algoritmos, se incorporan reglas, estándares e instrucciones que ayudan a estructurar las acciones de los usuarios en la infoesfera. La búsqueda, la adquisición y el manejo de todo tipo de información, a través de los dispositivos, redes sociales y medios digitales, tienen una influencia marcada en el comportamiento de los internautas.

El contexto digital no puede desligarse de la noción de los entornos sociales en los que la práctica de los usuarios tiene lugar en un contexto histórico, comunal y de un sentido cultural específico. Estos entornos abarcan no sólo los fenómenos mediáticos y sus procesos de apropiación, sino también los modos de hacer, discutir, representar y comunicar tendencias y normas sociales, así como patrones de comportamiento. Las nociones y construcciones sociales ocupan un lugar esencial en las redes sociales, pues al ser compartidas por una gran cantidad de internautas, sus significados se aceptan como una forma de decidir lo que se debe y no se debe hacer. En este sentido, se enfatiza el concepto de repetición (Schäfer, 2016) para tomar en cuenta lo cambios graduales y las diferencias que ocurren dentro de los entornos sociales. Desde un punto de vista praxeológico, el sociólogo Hilmar Schäfer reafirma la comprensión de la práctica como repetición que modifica aspectos esenciales de la vida social en términos de relaciones de poder, transformaciones históricas y diferenciación social. Por su parte, Theodore R. Schatzki (2016) plantea que los individuos y sus actividades, así como las estructuras y las instituciones, deben entenderse como productos, elementos o aspectos resultantes de la práctica o como un conjunto de acuerdos prácticos. Es decir, la relación entre prácticas y conjunto de acuerdos prácticos se analiza en términos de grosor, claridad y cohesión, de modo que los órdenes sociales no se entiendan como formaciones absolutamente cerradas, sino como dinámicas estables.

En las redes sociales se generan espacios para la participación cultural, política y comunicativa. Éstos se basan en prácticas aprendidas que se materializan desde y en el manejo de dispositivos y artefactos que traducen el comportamiento social a nuevos contextos, generando así procesos de formación de identidad. Los usuarios 
se definen a través de la manera en cómo se perciben a sí mismos en la red y el modo en cómo se integran a esos espacios sociales. Sus prácticas se enfocan en el registro y la descripción de procesos sociales que generan significados y espacios culturales con el fin de realizar operaciones básicas - desde el manejo de los Smartphones hasta la producción de contenido multimedia - en la producción y reproducción de abstracciones culturales. Quizás estas posibilidades técnicas lleven implícita la noción de que mediante su uso, muchos de los usuarios pueden desarrollar sus cualidades artísticas (fotografía, filmografía, danza, emprendimiento, obras de arte, entre otras), comunicativas (periodismo, blogs) y sociales (movimientos políticos, lucha por los derechos humanos, protestas).

A diferencia de la concepción de Martín Barbero antes expuesta, el sociólogo Andreas Reckwitz (2003), argumenta que la práctica consiste en ciertos movimientos y actividades rutinarias que no se limitan a la lectura, la escritura, el habla y, en todo caso, a navegar por Internet. La materialidad de la práctica implica la adquisición de conocimientos y la performatividad de la acción. Básicamente, se trata de la capacidad de los usuarios para asumir una responsabilidad en el entorno digital, en el desarrollo de sus habilidades mediáticas y la comprensión práctica de sus acciones en los espacios públicos y privados. Así, se define la práctica como un tipo de comportamiento que consiste en la interconexión de diversos elementos entre sí, desde las formas de actividades corporales y mentales, los artefactos y las cosas en sus diversos usos, hasta un conocimiento previo en forma de comprensión, knowhow (saber hacer), estados emocionales y puntos de vista.

A través del uso de las plataformas digitales se desvirtúa el sentido de lo público, mientras los hábitos de consumo de los usuarios se modifican constantemente. El espacio público se vuelve opaco y lejano, en tanto lo público se entiende como ese "lugar imaginario donde quisiéramos conjurar o controlar el riesgo de que todo esté permitido" (Canclini, 2020: 50). Un aspecto importante es destacar la capacidad de los usuarios para crear una amplia red de comunicación sin restricciones de tiempo y espacio con el uso de sus dispositivos móviles en los espacios públicos (Gwiazdzinski, 2015). Al utilizar la tecnología digital, estos espacios se reorganizan de manera sociotécnica, debido a que la percepción de nuestra realidad se percibe como una geografía generada por los medios. Las tecnologías digitales, así como los medios de comunicación globales deben comprenderse como geomedia (Döring y Thielmann, 2009), debido a que su uso está estrechamente conectado con ubicaciones físicas específicas. Es decir, el número creciente de dispositivos físicos (hardware) equipados con tecnologías de localización GPS, WLAN y RFID, así como la expansión del uso de tecnología geoweb (software cartográfico) son características esenciales del cambio sociotécnico que tiene lugar hoy en nuestras sociedades. Desde una perspectiva técnica, el uso de los geomedia es "independiente de la ubicación, pero su contenido depende de la ubicación” (Döring y Thielmann, 2009: 13). 
En la sociedad de plataformas las prácticas mediáticas se comprenden como aquellas que transforman e influye en la incorporación del conocimiento a la vida social en el tiempo y espacio de su realización. Los entornos digitales serían aquellas áreas relativamente limitadas pero nunca homogéneas de la vida social en los que se expresa la diversidad sociocultural en el uso de los medios digitales (Pink et al., 2016). Los procesos de mediatización de la vida cotidiana posibilitan una variedad de opciones para la participación, en tanto los consorcios de Internet estructuran el acceso, la oferta y la demanda, y los usuarios diseñan espacios sociales compartidos con nuevos significados. Este enfoque praxeológico investiga no sólo las prácticas mediáticas, sino también una compleja estructura de relaciones culturales, económicas y políticas, así como construcciones sociales (Couldry, 2004). Las prácticas mediáticas se entienden, además, dentro de un campo de tensión entre las rutinas de uso, la emergencia y la acción social. Éstas no solamente describen procedimientos no planificados y espontáneos, sino también coordinados, a largo plazo y estables que definen y condicionan el manejo de la tecnología (Dang-Anh et al., 2017). Este análisis se realiza teniendo en cuenta las propiedades físicas y sensoriales que determinan las materialidades de la comunicación en su difusión transcultural (Ginsburg et al., 2002).

\section{Capitalismo digital: interacciones y mercadotecnia}

El término sociedad de plataformas se define a partir del uso de los diversos formatos de medios digitales como modelos de negocio o empresarial que se caracterizan por proporcionar la infraestructura central para mediar la interacción entre las empresas (productores), diferentes grupos de usuarios, individuos interconectados entre sí, además de organizaciones e instituciones con bienes y servicios (Nash et al., 2017). La promesa de la adquisición de competencias mediáticas para la interacción que llevan implícita las plataformas se realiza como una especie de gadget o artilugio para atraer la atención de los consumidores/usuarios. El hecho de que las plataformas utilicen los datos suministrados por los usuarios, no implica que éstos sean considerados como individuos interconectados que dependan del control, la regulación y la manipulación de las compañías, pues en su mayoría, las interacciones sociales se ven modificadas por procesos de valorización y de negociación de sentido. En las plataformas digitales se genera una lógica propia que difiere de los modelos tradicionales de la comunicación, ya que ésta se diseña y reproduce como práctica a través del consumo de bienes y servicios de información y, en última instancia, como mercantilización de la práctica social mediante el procesamiento de datos. Así, se argumenta que los usuarios con sus acciones en el entorno digital, definen desde su 
consumo qué elementos forman parte de sus rutinas y prácticas, debido a que ellos también influyen en el modo y la frecuencia del uso de las plataformas.

La ya omnipresente crisis provocada por la Covid-19, ha agudizado las tendencias neoliberales en el manejo y el uso de las plataformas digitales. Estas tendencias describen y expresan procesos económicos muy dinámicos que van desde la descentralización y la privatización hasta modernización de la infraestructura tecnológica, los sistemas de información y las relaciones laborales. Desde principios del año 2020, se ha experimentado un proceso paulatino de recesión global donde las tasas de ganancias, así como la productividad y la eficiencia de muchas empresas en la rama de la industria se ven afectadas. No obstante, el comercio electrónico ha aumentado considerablemente. Los largos meses de cuarentena, la transición hacia las posibilidades que ofrece el teletrabajo, el distanciamiento social, las transacciones monetarias desde tarjetas de débito o crédito, las transferencias bancarias con la ayuda de servicios como PayPal, Klarna o Zelle, el aumento de las demandas de los consumidores mediante el envío de productos o la asistencia de servicios de entrega a domicilio, el paso de muchos modelos de negocio en el sector empresarial, autónomo y en el de los emprendedores hacia el entorno digital, son algunos ejemplos que ilustran el proceso creciente de digitalización de las actividades cotidianas.

El término capitalismo digital (Schiller, 2014) hace referencia a un nuevo orden económico en el que las plataformas digitales se articulan como intermediarios para estructurar y regular la oferta y la demanda en los diversos mercados que conforman el Internet. Estos mercados se transforman en metaplataformas propietarias que controlan la distribución de la riqueza social, el acceso a los bienes y servicios, además de estructurar el trabajo y los modelos de negocio que ellas utilizan (Busch, 2021, Dolata, 2020). El mercado digital puede definirse, como ese lugar que abarca un conjunto de procesos, rutinas, hábitos y estrategias de mercadotecnia que responden a la competencia entre los grandes consorcios de Internet a escala global, en el que la adquisición y el procesamiento de datos son la materia prima para expandir los modelos de negocio y aumentar las ganancias. Los datos originados, producidos y puestos a disposición en las plataformas digitales por los usuarios, a través de redes de trabajo, consumo, ocio y entretenimiento, amistad, entre otras, se manipulan, se empaquetan y se comercializan como mercancías (Srnicek, 2018).

Los cambios que trae consigo el capitalismo digital están marcados por cuatro estrategias fundamentales: 1) el control de la información con el objetivo de la recepción exclusiva de datos de mercado, 2) el poder que implica la adquisición de la información permite el control efectivo del acceso al propio mercado, donde no sólo se abre una nueva área de negocios diferenciada, sino que, 3) una estrategia atractiva y eficaz para controlar los precios, 4) desarrollando un sistema de control para las operaciones e indicadores de desempeño para productores y operadores dentro 
del mercado de datos con el fin de dictar las condiciones, en forma detallada, para la prestación de los más diversos servicios (Staab, 2019). La interfaz de usuario, los sistemas de seguimiento (tracking) y de calificaciones (ratings) serían algunos de los nuevos métodos o herramientas para el control de la gestión de la información con los que se supervisa, vigila y regula todo el flujo de trabajo. A estos procesos se vinculan también sanciones, por ejemplo, si los consumidores/usuarios no cumplen con las reglas y normas de uso de alguna plataforma, los operadores pueden bloquear el acceso o cancelar el servicio prestado.

Desde la década de los años 2000, las sociedades contemporáneas actuales vienen experimentando un proceso de digitalización de su economía. Mediante sus estrategias de innovación y, fundamentalmente, a través de los proyectos de investigación y desarrollo, las empresas tecnológicas han experimentado un desarrollo significativo en términos de poder político y capital financiero alcanzando un rol determinante en la mediación de todo tipo de relaciones sociales en el entorno digital. En el contexto del auge de la digitalización y automatización en la denominada Industria 4.0 ${ }^{1}$, en la aplicación de algoritmos para el control del uso de los dispositivos digitales y el Internet (Cardon, 2018), así como en la cibernetización de las actividades cotidianas (Kaerlein, 2018), surge la también denominada economía de plataformas que demanda, dentro de los procesos de innovación de las empresas de Internet, un cambio tecnológico constante. Estas transformaciones sociotécnicas implican estrategias para reducir costos, eliminar la competencia, reducir los tiempos muertos, ganar participación en los mercados, rediseñar continuamente los procesos productivos y los flujos de trabajo (Pérez Martirena, 2020, Canclini, 2020). Estos procesos son fuente del dinamismo del capitalismo, debido a que los capitalistas tienden a incrementar constantemente la productividad laboral y a superarse unos a otros en la manera de generar ganancias de modo eficiente. Así, la tecnología cumple un papel esencial, pues "(...) es utilizada con frecuencia para despojar de calificaciones a los trabajadores y socavar el poder los empleados calificados" (Srnicek, 2018: 17-18).

Las plataformas digitales se constituyen como actores clave en la transformación digital y son impulsoras del crecimiento económico, en tanto promueven un cierto tipo de desarrollo social al convertirse en intermediarios en diversos sectores de la sociedad. Esta transformación sociotécnica incluye no sólo sectores tradicionales como el manufacturero, el de servicios $y$ transporte $o$ el de las

${ }^{1}$ Ver la entrevista con uno de los fundadores del término "Industria 4.0", el profesor Wolfgang Whastler en: Deutsches Forschungszentrum für Künstliche Intelligenz (2020). Prof. Wolfgang Wahlster on Industrie 4.o. Recuperado de https://ricaip.eu/prof-wolfgang-wahlster-on-industrie-4o/. Para la publicación original en idioma alemán ver: VDI Nachrichten (2011). Industrie 4.0: Mit dem Internet der Dinge auf dem Weg zur 4. industriellen Revolution. Recuperado de https://www.dfki.de/fileadmin/user_upload/DFKI/Medien/News_Media/Presse/Presse-Highlights/vdinach2011a13-ind4.o-Internet-Dinge.pdf. 
telecomunicaciones, sino que comienza a abarcar infraestructuras de servicios de asistencia social. En este sentido, las grandes plataformas digitales no sólo controlan los mercados, sino también infraestructuras económicas y sociales centrales, en tanto penetran cada vez más en el ámbito de la atención primaria con la prestación de servicios fundamentales como la movilidad, la salud y la educación. A través del avance rápido de la plataformatización en las áreas que forman parte de la prestación de servicios básicos para los ciudadanos en la sociedad digital, se pueden divisar amenazas que perturban el ámbito de los servicios de interés general. Los proveedores estatales y municipales de servicios de asistencia social dependen cada vez más de las plataformas digitales. Las instituciones legitimadas democráticamente van perdiendo el control de la prestación de servicios de asistencia social garantizados por el Estado. A esto se le suma, que “(...) algunas prestaciones de servicios digitales mediadas por las plataformas se han vuelto tan indispensables desde el punto de vista social, que estos mismos podrían considerarse parte de la provisión de servicios digitales de asistencia social" (Busch, 2021: 6-7).

En las plataformas digitales, la comunicación tiene como objetivo establecer conexiones entre individuos, dispositivos y artefactos, entre programas informáticos y usuarios finales para el intercambio de datos e información, ideas, emociones, puntos de vista u opiniones mediante códigos compartidos en espacios comunes. Las plataformas, además, “(...) se posicionan como intermediarias que reúnen a diferentes usuarios: clientes, anunciantes, proveedores de servicios, productores, distribuidores e incluso a objetos fisicos" (Srnicek, 2018: 45) para mediar todas las relaciones sociales que se establecen en la infoesfera. Su funcionamiento tiene como base un concepto amplio, y de carácter social, de infraestructura que incluye tanto fundamentos materiales como inmateriales. El término infraestructura, aquí no se limita sólo a aquellos fundamentos materiales - tráfico, comunicaciones o suministro de energía y de agua -, que forman el núcleo de este concepto, sino la atención recae en la importancia que tienen las infraestructuras en la sociedad digital para establecer los requisitos previos hacia la satisfacción de las necesidades básicas y para conformar la participación social. Desde el punto de vista del debate actual en los países europeos sobre el tema de la regulación gubernamental de las plataformas, se expresa que:

La regulación de las plataformas no es sólo una cuestión de la política de competencia, sino también una que le atañe a la política de infraestructuras y a la del suministro de servicios públicos. Por lo tanto, una regulación adecuada de las plataformas digitales no debe considerarlas sólo como actores/agentes del mercado ni orientarse sólo a entender las consecuencias económicas de una competencia limitada, sino debe tener en cuenta, además, su papel como infraestructuras centrales en la sociedad digital. De igual forma, se hace necesario examinar críticamente la creciente influencia de las plataformas digitales en el ámbito de la prestación de 
servicios públicos básicos. Ésto debe garantizar que las instituciones legitimadas democráticamente no transfieran [o entreguen] el control de las prestaciones esenciales de estos servicios de interés general a la economía de las plataformas (Busch, 2021: 7).

En este aspecto se hace necesario señalar que las estrategias de innovación, la digitalización de los procesos productivos y las prácticas de mercadotecnia de los consorcios de Internet conllevan a la simplificación de tareas y brindan, hasta cierto punto, facilidades a los usuarios. Por ejemplo, realizar un trabajo desde un espacio físico distinto al de la oficina, o sea, en el hogar (teletrabajo) o en el espacio público a través de plataformas como Uber o de entrega de productos a domicilio. Estas prácticas implican una dependencia a tecnologías cada vez más complejas que se almacenan en dispositivos técnicos como los Smartphones. Los teléfonos inteligentes ocupan un lugar central en la vida contemporánea, ya que son un elemento esencial tanto para el consumo cultural, el ocio, el entretenimiento y la comunicación social, así como para la búsqueda de información y de trabajo, para estructurar y organizar la jornada laboral de muchos empleados a tiempo parcial. Esta situación permite un nuevo tipo de explotación de la fuerza de trabajo, debido a que el proceso laboral se hace cada vez más inmaterial y se orienta hacia el uso y la manipulación de símbolos, las emociones y las perspectivas de futuro de los usuarios (Féliz y Bona, 2020).

En este sentido, las plataformas digitales son el lugar para la producción de ganancias como valor agregado de los datos que los usuarios producen sin costo alguno. Ellas regulan y controlan, a través del uso de algoritmos, los motores de búsqueda, la información de los perfiles, las fotos, los videos, la actualización de estados y el contenido de las publicaciones de millones de usuarios. Google, por ejemplo, controla el acceso de miles de millones de personas al conocimiento, lo que lo ha convertido en un factor indispensable para la formación de opiniones públicas o en un elemento que influencia la voluntad política en las democracias occidentales modernas. El carácter indispensable de este servicio de búsqueda de información, tan esencial para la sociedad, justifica el derecho del Estado a imponer al prestador de servicios determinadas obligaciones de interés general (Busch, 2021). Otro ejemplo aquí serían plataformas como Facebook, Twitter, Instagram y Tik Tok en su función de agenda setting, es decir en la conformación, el tratamiento y la difusión de temas específicos, tendencias, puntos de vista, opiniones y modos de hacer que modifican el consumo y las acciones de los usuarios. Éstas han alcanzado una relevancia en la producción de la opinión pública dentro de las redes sociales, debido a que estructuran no sólo los espacios de acción social en el entorno digital, sino que transforman esos espacios virtuales en infraestructuras centrales para la participación política y cultural de la sociedad civil, en tanto permiten la inclusión social, pero también la discriminación, la exclusión, la intolerancia, entre otros aspectos. De esta 
forma, las plataformas generan "los fundamentos de una sociabilidad constituida desde el sector privado de la economía en Internet" (Dolata, 2020: 17).

\section{Paradigmas: regulación e interoperabilidad}

De manera aforística y casi paradigmática, Marshall McLuhan (1994) consideraba a los medios como entornos, en tanto se constituyen como metáforas activas en su poder de traducir las experiencias en nuevas formas. Manuel Castells (2002) precisaba que el nuevo paradigma tecnológico que se introduce con la revolución provocada por el Internet, se constituye de dos expresiones fundamentales. Primero, a través de Internet se establecen nuevas pautas para la producción de conocimiento y el procesamiento de la información. Precisamente, mediante el uso de las tecnologías de la información no se determinan los procesos de generación de conocimiento o de tratamiento de datos, sino que la tecnología se desarrolla en relación con contextos sociales, institucionales, económicos y culturales. Segundo, es la capacidad que tiene el uso de la tecnología en su poder de recodificar la materia viva, en tanto todos los procesos de la sociedad, de la política, de la guerra, de la economía pasan a verse afectados por la capacidad de procesar y distribuir energía de forma ubicua en el conjunto de la actividad humana.

Uno de los elementos que en la actualidad modifica este paradigma cultural del uso de Internet es el factor económico, ya que los consorcios de Internet poseen la propiedad sobre el activo más importante en la red, la plataforma de software y el análisis de datos. Este derecho económico de propiedad, además de presuponer la posesión de los activos financieros en términos de liquidez, rentabilidad e inversiones de riesgo, implica la transición del paradigma de la industria al de la prestación de servicios. Para Srnicek (2018), este proceso se agudiza con la burbuja puntocom entre los años 1997 y 2001, durante los cuales se produjo un crecimiento acelerado de los valores económicos de muchas empresas en los países industrializados, vinculadas principalmente al sector de Internet y su nueva economía. El análisis de datos es el factor determinante para la selección, adquisición y almacenamiento de las informaciones de los perfiles de los usuarios como productos comercializables. La "centralidad del análisis de datos les permite a las plataformas operar con una escasa cantidad de activos y funcionar únicamente como vía de encuentro entre diversos actores" (Combina y Paltrinieri, 2020: 7).

Las plataformas digitales se constituyen como agentes económicos, políticos e institucionales de la transformación sociotécnica en tanto se definan en términos de organización, poder y cálculo (Peters, 2015). Al basar su funcionamiento en la minería de datos, los consorcios de Internet construyen una realidad informática generada mediante el tratamiento, el procesamiento y el almacenamiento de una gran 
cantidad de datos. Estos procesos mediáticos determinan el significado de la apropiación y la percepción sensorial de los usuarios, haciendo que todos y cada uno de los procesos de mediatización sean visibles en términos de datos construidos y calculados. En este sentido, se afirma que a través de las plataformas digitales se genera una cultura organizacional como ese conjunto de ideas y concepciones, prácticas y valores, reglas y normas que le son comunes tanto a las empresas como a sus usuarios, pues las acciones de las empresas se materializan en relaciones económicas, políticas y culturales. Una de las premisas para esta lógica digital de organización, es que todo lo que se escriba, se piense, se describa y establezca relaciones de dependencia, pueda expresarse, reducirse y calcularse dentro del código binario o y 1 (Peters, 2015). En el momento que esta lógica se orienta al mundo, se vuelve operativa y desarrolla sus poderes organizativos, debido a que "cuando la realidad es indexada por una máquina digital, los datos resultantes se procesan y retroalimentan al mundo como un conjunto de observaciones o instrucciones (...)" (Beverungen et al., 2019: 627).

La materialidad de la comunicación, se refiere aquí a esa base técnica que posibilita el desarrollo de habilidades mediáticas y se correlaciona con las rutinas de uso. De igual manera, se afirma que estas rutinas homogeneizan las diversas prácticas culturales y mediáticas de los usuarios, condicionando sus acciones, negociaciones de sentido y hábitos de consumo en el entorno digital. Las prácticas mediáticas aquí son el modo en que los usuarios producen de manera suficiente diversos recursos digitales como información y datos, y las empresas los transforman en productos y servicios. Las prácticas mediáticas están en dependencia directa con las infraestructuras técnicas puestas a disposición por las plataformas digitales y se constituyen y modifican a sí mismas el contexto de su realización. No obstante, desde las infraestructuras se generan las prácticas mediáticas en dos puntos esenciales. En primer lugar, las infraestructuras están sujetas a una forma específica de transparencia, en tanto sean visibles a primera vista o sean completamente invisibles. En segundo lugar, en términos de infraestructura, las prácticas mediáticas son materiales o inmateriales, se distribuyen local o globalmente, o son estáticas o dinámicas, pues requieren ciertas infraestructuras para su funcionamiento (Dang-Anh et al., 2017).

Desde una perspectiva cultural, se puede argumentar que las plataformas digitales generan, en lo referente a la organización, centros de poder, ejes temáticos que orientan redes de discurso, un conjunto de acuerdos prácticos que determinan las prácticas mediáticas y agrupaciones sociales que comparten espacios públicos y privados. Pues, los medios funcionan como "dispositivos de ordenamiento de civilizaciones" (Peters, 2015: 5) o "materiales para administrar el tiempo, el espacio y el poder" (Peters, 2015: 20). Esta determinación se orienta hacia una reflexión del condicionamiento, la estructuración material y tecnológica de nuestra experiencia con los agenciamientos y la interacción que posibilitan las plataformas digitales. 
La transformación de la dimensión cultural de Internet se sustenta, para los fines de este artículo, en varios aspectos. Por un lado, las plataformas potencian la idea del emprendimiento como elemento regulador de las relaciones e interacciones entre las empresas y sus usuarios. Plataformas como Airbnb, Uber y las de entrega de productos a domicilio como Deliveroo más que potenciar sus productos, facilitan la prestación de servicios. Estos modelos de negocio generan un tipo de contratación tercerizada, donde se oculta la relación entre trabajo y capital, además de que se publicitan a ellas mismas como meros puntos de encuentro entre los actores o agentes que interactúan entre sí (Scasserra, 2016). Por otro lado, se trastoca la idea de la relación entre iguales, en tanto las informaciones y los datos se adquieren, almacenan y analizan mediante el empleo de algoritmos que le pertenecen como propiedad privada a las empresas. Los algoritmos no son entidades neutrales capaces de eliminar del entorno digital fenómenos tales como el sexismo, el racismo, la homofobia, entre otros, sino que reproducen los roles de género, regulan la conducta a la hora de expresar opiniones y potencian la idea falsa del anonimato en el uso de las redes sociales.

Los algoritmos cumplen una función específica, debido a que implementan las reglas y normas sociales que se aplican en las plataformas como instrucciones técnicas, ya que monitorean y sancionan las actividades de los participantes y deciden, con base en los criterios de relevancia social escritos en ellos, sobre lo que es importante y lo que no lo es. Ellos seleccionan, agregan y clasifican la información, las noticias, los videos o fotos, y estructuran los procesos de información y comunicación privada, así como las posibilidades de discurso público, contribuyendo a la formación de públicos y comunidades que no existirían sin ellos. De este modo, “(...) se convierten en el núcleo de un marco mediado técnicamente hacia el control de la acción social" (Dolata, 2020: 19-20). Un aspecto paradójico aquí es que los consorcios de Internet no se encuentran fuera de la sociedad como entes externos que sólo supervisan, vigilan y controlan, sino que son partícipes en las intervenciones políticas y estatales, en los intereses de otros actores y agentes económicos, en las protestas de algunos grupos de la sociedad civil y el comportamiento de los usuarios hacia la conformación de gustos, deseos, tendencias y del consumo. Las plataformas digitales se constituyen a sí mismas como actores decisivos y proactivos no sólo en el diseño y la estructuración de un marco normativo para el funcionamiento de Internet, sino también en los procesos de toma de decisiones políticas. Para el sociólogo Ulrich Dolata (2020), los algoritmos, entendidos como el fundamento de toda búsqueda e información, comunicación e interacción en estas plataformas, son programas altamente políticos que construyen ofertas diferenciadas, selectivas y cada vez más personalizadas de la realidad social a partir de criterios absolutamente ambiguos que se aplican tanto a individuos como a organizaciones y a la política. 
Otro elemento técnico que posibilita la estructuración de los espacios de acción social para habilitar y canalizar las actividades de los internautas, es la interfaz de usuario. Ésta se personaliza y configura en correspondencia con los estándares de las plataformas, con el objetivo de influir en las acciones de los usuarios, al permitir ciertas actividades y excluir otras. A través de la interfaz de programación de aplicaciones (Application Programming Interface - API, por sus siglas en inglés), muchos sitios web de terceros se han integrado a las acciones de las plataformas digitales dominantes. Así se establecen amplios enlaces entre éstas y los sitios web externos, para permitir la comunicación entre dos sistemas diferentes y extender el rango de acción en términos de función, adquisición de datos y el uso de aplicaciones. El control y la regulación de la interfaz de usuario posibilita que su influencia se extienda mucho más allá del dominio original de una plataforma, abarcando otros sitios web e influyendo en el campo de acción de otros actores en la red. La integración de terceros permite a los operadores de las plataformas dominantes desviar sistemáticamente la información y los datos, dando como resultado que éstos se acumulen, utilicen, recopilen y se analicen para cumplir sus propios fines.

Por este motivo, se habla hoy sobre una plataformatización (Busch, 2021) de la sociedad, en tanto se delimitan en sectores determinados, y de forma significativa, las arquitecturas técnicas provocando que el alcance de las plataformas y su importancia social vaya en ascenso. En este contexto, las prácticas de los usuarios se generan dentro de relaciones asimétricas caracterizadas por procesos de concentración del poder y control de la acción social. En los procesos de organización y circulación de la actual economía digital se coordinan contextos de trabajo y se controlan las actividades de los usuarios, en tanto las contribuciones de los productores $\mathrm{u}$ operadores de red posibilitan la creación de valor agregado en torno a los contenidos que se consumen, mercantilizando las diversas formas de la comunicación. Las plataformas digitales son infraestructuras sociotécnicas que basan su funcionamiento en el procesamiento de datos y en el uso de algoritmos para intercambiar información, estructurar la comunicación y la producción, organizar el trabajo y los mercados, además de ofrecer una amplia gama de servicios o vender productos digitales y no digitales (Srnicek, 2018). No obstante, se puede argumentar que el éxito o fracaso de las plataformas depende, en cierta medida, de la cantidad de usuarios, de sus contribuciones personales en términos de reproducción de los contenidos, de las comunicaciones e interacciones que ellos establecen, además de sus evaluaciones y preferencias. 


\subsection{Normativas para la regulación de las plataformas digitales}

En la esfera de la política, la economía y la cultura, la actividad comercial implícita en las plataformas digitales le impone nuevos retos a la protección de la privacidad y al almacenamiento los datos personales. Por este motivo y luego de varios años de debate (Gorjón, 2020, Vilalta Nicuesa, 2018), se promulgó a finales del año 2020 una nueva norma jurídica - Reglamento del Parlamento Europeo y del Consejo de la Unión Europea - para la regulación de un mercado único, a través de la propuesta de Ley de Servicios Digitales (Digital Services Act) que modifica la anterior Directiva $2000 / 31 / \mathrm{CE}^{2}$. El objetivo de este reglamento es refrendar un nuevo marco legal con pautas y directrices establecidas para el funcionamiento del mercado digital, incluidas las plataformas digitales, y para garantizar un entorno digital más seguro para los usuarios y las empresas en toda la Unión Europea.

Esta norma jurídica establece un conjunto común de reglas o preceptos sobre las obligaciones de los intermediarios en las plataformas digitales, además de regular la rendición de cuentas en todo el mercado único europeo. De esta manera se pretenden abrir otras oportunidades para la prestación de servicios digitales a través de las fronteras, garantizando un alto nivel en la protección de los datos personales de los usuarios, con independencia del lugar donde residan dentro de la Unión Europea. Los objetivos de esta normativa incluyen: 1) Regular lo digital y establecer las pautas que deberán cumplir las grandes empresas tecnológicas, desde la publicidad y la gestión de los datos hasta la responsabilidad de las plataformas por el contenido que se publica en ellas. 2) Renovar la directiva del comercio electrónico. 3) Definir la responsabilidad de las plataformas sobre el contenido que se publica en ellas y crear la figura de gatekeeper (controladores), para deslindar aquellas que cuentan con demasiada influencia en el mercado. 4) Diferenciar las normas que deben aplicarse al contenido normal y cuáles deben adjudicarse a los contenidos patrocinados y otras publicaciones publicitarias, aclarando así el concepto de contenido ilegal y estableciendo qué contenido es de carácter económico y lo que no lo es. 5) Abarcar un amplio espectro de prácticas mediáticas desde las redes sociales, hasta los servicios de la economía colaborativa, pasando por los motores de búsqueda, la publicidad en línea, los servicios en la nube, los puntos de acceso wifi, el alojamiento de páginas web, los servicios de mensajería, las tiendas de aplicaciones, la prestación

\footnotetext{
${ }^{2}$ Para más información sobre la Ley de Servicios Digitales ver el sitio web https://ec.europa.eu/info/strategy/priorities-2019-2024/europe-fit-digital-age/digital-services-act-ensuringsafe-and-accountable-online-environment_es. La Directiva 2000/31/CE puede descargarse en

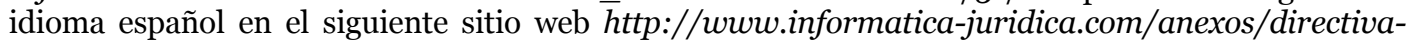
20oo-31-ce-del-parlamento-europeo-y-del-consejo-de-8-de-junio-de-20oo-relativa-a-determinados-aspectos-juridicos-de-los-servicios-de-la-sociedad-de-la-informacion-en-particular-el-comercio-e/.
} 
de servicios basados en la inteligencia artificial, las redes de suministro de contenidos y los servicios de nombres de dominio.

La plataformatización de la actividad comercial respresenta una transformación sociotécnica muy ambivalente en la arquitectura y la infraestructura del mercado digital actual. Las plataformas de comercio electrónico reducen los costos de transacción y aumentan la eficiencia del intercambio de mercancías. Los operadores de plataformas adoptan, además, la interfaz de usuario como mecanismo para integrarse en la relación entre proveedores y demandantes, coordinando la convergencia entre la oferta y la demanda. La gestión del mercado mediante sistemas algorítmicos se realiza de forma centralizada, en tanto los operadores de plataformas asumen el rol de legisladores privados con capacidad proactiva, promulgando las normas y reglas para la participación en el intercambio de los más diversos servicios (Busch, 2021).

La interoperabilidad es otro modelo que se propone para regular la actividad comercial de la plataformas digitales. Este término hace referencia a la posibilidad de que los usuarios de servicios de mensajería instantánea y llamadas, o sea, aplicaciones que utilizan software libres de código abierto que hacen énfasis en la seguridad y la protección de la privacidad, puedan comunicarse con usuarios que utilizan otras aplicaciones. De esta manera, tendrían la opción de elegir un proveedor como plataforma de acceso, por ejemplo, Telegram, y desde esta aplicación, comunicarse con los usuarios de todas las demás plataformas.

Con este modelo, las plataformas de redes sociales se transformarían en puntos de acceso a una infraestructura de comunicación descentralizada en la que se limitan la manipulación del consumidor, el aumento de la competencia, la especulación y la publicidad no deseada. Las barreras para el acceso al mercado de proveedores nuevos más pequeños se reducirían, en tanto se establece una competencia menor en el sistema de gestión de la información y las comunicaciones, así como en las tendencias al monopolio de los datos. La interoperabilidad permitiría que los usuarios de redes sociales pueden seleccionar un proveedor de plataforma en el que confíen o que se base en el uso de softwares de código abierto. De este modo, los usuarios podrían generar sus propios puntos de acceso a través del cual puedan administrar, controlar y proteger el flujo de datos dentro del sistema de información en el que se encuentren. El uso de diversas plataformas, le permitiría a los usuarios defenderse contra la vigilancia, el control y la supervisión masiva de los datos que ingresa en la red. Con el modelo de la interoperabilidad, se evitarían los efectos nocivos que se generan desde rastreo del historial del navegador, el uso de cookies, el acceso a los contactos de los Smartphones y la geolocalización. Como resultado, esto dificultaría la microfocalización, el tracking, la manipulación de la información y los datos que los consorcios de Internet adquieren de los usuarios (Piétron, 2019). 
Aunque la interoperabilidad represente un modelo idealizado de un sistema de información más amigable para los usuarios, su aplicación sólo debe materializarse como componente esencial de la regulación integral de las plataformas digitales. La transformación sociotécnica de Internet, que las empresas implementan mediante el uso de tecnologías cada vez más complejas, imponen tendencias y modelos de negocio que se hacen imposibles de regular y controlar. Empresas como Google, Amazon, Facebook y Apple desarrollan a un ritmo vertiginoso mecanismos innovadores con nuevas funcionalidades que les permiten reestructurar y afianzar su posición en el mercado digital. Ellas poseen una posición privilegiada en lo referente al acceso, la adquisición y el almacenamiento de las informaciones y los datos de los usuarios, además de disponer de suficiente capital financiero para retener a los consumidores y aumentar los costos de los servicios de marketing en términos de publicidad. En términos de la distorsión que esto genera dentro del mercado, la respuesta a la regulación de esta actividad comercial quizás sólo sea asunto de la política estatal y la promulgación de normativas, regulaciones y leyes que limiten el poder de las corporaciones, sobre todo, cuando las plataformas digitales influyen y modifican de manera creciente la infraestructura social. Desde un punto de vista social, las plataformas están sujetas a la supervisión pública, ya que “(...) las intervenciones del Estado no sólo deben enfocarse en combatir el abuso de poder en el mercado digital o en el incumplimiento de las normas de protección de datos, sino que también deben orientarse a mejorar continuamente la autodeterminación informativa y la soberanía digital de los ciudadanos frente al cambio tecnológico" (Piétron, 2019: 4).

Las transformaciones constantes en los procesos de innovación de los consorcios de Internet constituyen elementos fundamentales para el desarrollo de una sensibilidad colectiva cambiante. Comprender la densidad de estas transformaciones sociotécnicas modifica los modos de comunicar en términos de una experiencia cultural nueva en las formas de percibir, interactuar y responder a los cambios en el entorno digital. A partir de la socialidad técnica que se establece en y desde las plataformas digitales, se puede argumentar que los mecanismos usados por los consorcios de Internet se centran, en primer lugar, en la organización y regulación de los mercados mediante el sector privado. Los operadores de plataforma coordinan los procesos y estrategias, determinando las condiciones competitivas. En segundo lugar, las tecnologías cada vez más complejas que se utilizan, estructuran las condiciones materiales y sociales, en tanto el comportamiento de los usuarios se regula mediante funciones de orden social e institucional. Gran parte del intercambio privado y público de información, así como la mayoría de las actividades económicas en Internet, se desarrollan hoy en espacios organizados y diseñados de forma privada y dentro de los marcos técnicos y socioeconómicos establecidos por las empresas que ofrecen las ofertas, productos y servicios correspondientes (Dolata, 2020). 


\section{Conclusiones}

La cultura organizacional que se genera desde el uso de las plataformas digitales se centra en la estructuración de proyectos de infraestructuras digitales que determinan las materialidades de la comunicación. Estas infraestructuras, a menudo ocultas en los dispositivos técnicos - mediante el tratamiento y procesamiento de datos, el uso de códigos y algoritmos -, y en las que el poder y la gobernabilidad están integrados y materializados, implican el diseño de nuevos mecanismos de control por parte de los consorcios de Internet y una transformación de las prácticas mediáticas de los usuarios. No obstante, existe una profunda brecha entre el accionar de las plataformas, las instituciones reguladoras de los procesos en red y los consumidores/usuarios que utilizan y coproducen los medios digitales. Esta brecha se define, principalmente, desde la forma en cómo se actúa y se percibe el espacio para la interacción, además de cómo se producen y almacenan los datos, la información, y sobre todo, el conocimiento.

Las interacciones que se establecen en y desde las plataformas modifican las expresiones digitales de los más diversos actores sociales (usuarios) que actúan en la red, mientras éstos se relacionan con artefactos, productos y servicios. La sociedad de plataformas se orienta por los intereses económicos, políticos y culturales de las empresas, que pretenden, por medio de diferentes estrategias de mercadotecnia y procesos de innovación, desarrollar, producir y dar forma a las culturas digitales. Pues, cada plataforma pretende apropiarse de un espacio social, de un nicho dentro del mercado digital, para organizar, controlar y regular de manera sociotécnica, el funcionamiento de sus sistemas de información. Un elemento esencial es que a través de las infraestructuras técnicas, se genera una economía organizada de forma privada y corporativa que controla los mercados digitales mediante la oferta y la demanda, regulando la actividad de los usuarios y determinando el uso diario de la tecnología en todas las esferas de la vida social. Pensar estas transformaciones en términos de mediación no se dirige a analizar lo que se representa en y por los medios, sino en reflexionar sobre las condiciones materiales y técnicas que hacen posible visualizar, materializar y representar la percepción sensorial y lo que éstas significan para nuestra experiencia cultural y lo que hacemos dentro de los entornos sociales que construimos. 


\section{Bibliografía}

Appadurai, A. (1996). Modernity at Large: Cultural Dimensions of Globalization. Minneapolis: University of Minnesota Press.

Beverungen, A., Beyes, T., Conrad, L. (2019). The organizational powers of (digital) media. Organization, 26(5), 621-635. https://doi.org/10.1177/1350508419867206

Busch, C. (2021). Regulierung digitaler Plattformen als Infrastrukturen der Daseinsvorsorge. Friedrich Ebert Stiftung, en https://library.fes.de/pdf-files/wiso/17527.pdf.

Cardon, D. (2018). Con qué sueñan los algoritmos. Nuestras vidas en el tiempo de los Big Data. Madrid: Dado Ediciones.

Castells, M. (2002). La dimensión cultural de Internet. Institut de cultura: Debates culturales, en https://www.uoc.edu/culturaxxi/esp/articles/castells0502/castellso502.html.

Combina, F., Paltrinieri, M. (2020). Plataformas austeras. Conflictos y posibilidades en la economía actual. En M. Féliz y L. Bona (Ed.). (2020). Economía de Plataformas. Problemas, debates y casos de estudio. La Plata: Universidad Nacional de La Plata.

Couldry, N. (2004). Theorising media as practice. Social Semiotics 14(2), 115-132.

Dang-Anh, M., Pfeifer, S., Reisner, C., Villioth, L. (Ed.) (2017). Medienpraktiken. Situieren, erforschen, reflektieren. Navigationen. Zeitschrift für Medien- und Kulturwissenschaften, 17 (1), 7-36.

Dolata, U. (2020). Plattform-Regulierung. Koordination von Märkten und Kuratierung von Sozialität im Internet. Berliner Journale für Soziologie 29, 179-206.

Dolata, U., Schrape, J. (2018). Kollektivität und Macht im Internet. Soziale Bewegung - Open Source Communities - Internetkonzerne. Wiesbaden: Springer.

Döring, J., Thielmann, T. (Ed.) (2009). Mediengeographie. Theorie - Analyse Diskussion. Bielefeld: Transcript.

Féliz, M., Bona, L. (Ed.) (2020). Economía de Plataformas. Problemas, debates y casos de estudio. La Plata: Universidad Nacional de La Plata.

Fernández, J. L. (2018). Plataformas Mediáticas. Elementos de análisis y diseño de nuevas experiencias. Buenos Aires: La Crujía.

Floridi, L. (2015). Die 4. Revolution. Wie die Infosphäre unser leben verändert. Berlin: Suhrkamp.

García Canclini, N. (2020). Ciudadanos reemplazados por algoritmos. Bielefeld: Calas.

Ginsburg, F., Abu-Lughod, L., Larkin, B. (2002). Media Worlds: Anthropology on New Terrain. Berkeley/Los Angeles: University of California Press. 
Gorjón, S. (2020). Plataformas digitales: avances en su regulación y retos en el ámbito financiero. Boletín Económico, Banco de España, issue 4/2020, 1-14, en https://ideas.repec.org/a/bde/joures/y2020i12daan33.html.

Gwiazdzinski, Luc (2015). The Urban Night: a Space Time for Innovation and Sustainable Development. Journal of Urban Research 11, 1-15.

Günzel, S. (Ed.) (2010): Raum: Ein interdisziplinäres Handbuch. Stuttgart/Weimar: J. B. Metzler.

Kaerlein, T. (2018). Smartphones als digitale Nahkörpertechnologien. Zur Kybernetisierung des Alltags. Bielefeld: Transcript.

Martín Barbero, J. (2010). Jóvenes: entre la ciudad letrada y el mundo digital. En G. Lluch (Ed.) (2010). Las lecturas de los jóvenes. Un nuevo lector para un nuevo siglo. Barcelona: Anthropos.

McLuhan, M. (1994). Understanding Media: The Extensions of Man [Orig. 1964]. Cambridge: MIT Press.

Nash, V., Bright, J., Margetts, H. Lehdonvirta, V. (2017). Public Policy in the Platform Society. Policy and Internet 9 (3-4), 368-373.

Pérez Martirena, S. (2020). Hacia una comprensión del capitalismo plataformas: un análisis de caso acerca de Glovo. En M. Féliz y L. Bona (Ed.). (2020). Economía de Plataformas. Problemas, debates y casos de estudio. La Plata: Universidad Nacional de La Plata.

Peters, J. D. (2015). The Marvelous Clouds. Toward a Philosophy of Elemental Media. Chicago: University of Chicago Press.

Piétron, D. (2019). Digitale Souveränität durch Interoperabilität. Zur Möglichkeit dezentraler sozialer Netzwerke in der Plattformökonomie. Friedrich Ebert Stiftung, en http://library.fes.de/pdf-files/wiso/15852.pdf.

Pink, S., Horst, H., Postil, J., Hjorth, L., Lewis, T., Tacchi, J. (2016). Digital Ethnography: Principles and Practice. London: Sage.

Reckwitz, A.(2003). Grundelemente einer Theorie sozialer Praktiken. Zeitschrift für Soziologie 32 (4), 282-301.

Scasserra, S. (2016). Las plataformas web (y que demandar desde el sindicalismo latinoamericano). Friedrich Ebert Stiftug, en https://library.fes.de/pdf-files/bueros/uruguay/14695.pdf.

Schatzki, T. R. (2016). Praxistheorie als flache Ontologie. En H. Schäfer (Ed.) (2016). Praxistheorie. Ein soziologisches Forschungsprogramm. Bielefeld: Transcript.

Schäfer, H. (2016). Praxis als Wiederholung. Das Denken der Iterabilität und seine Konsequenzen für die Methodologie praxeologischer Forschung. En H. Schäfer (Ed.) (2016). Praxistheorie. Ein soziologisches Forschungsprogramm. Bielefeld: Transcript. 
Schiller, D. (2014). Digital Depression. Information Technology and Economic Crisis. Illinois: University of Illinois Press.

Silverstone, R., Hirsch, E. (1992). Consuming Technologies: Media and Information in Domestic Spaces. London/NewYork: Routledge.

Srnicek, N. (2018). Capitalismo de plataformas. Buenos Aires: Caja Negra.

Staab, P. (2019). Digitaler Kapitalismus: Markt und Herrschaft in der Ökonomie der Unknappheit. Berlin: Suhrkamp.

Stalder, F. (2016). Kultur der Digitalität. Berlin: Suhrkamp.

van Dijck, J., Poell, T., de Waal, M. (2018). The Platform Society: Public Values in a Connective World. Oxford: Oxford University Press.

Vilalta Nicuesa, A. E. (2018). La regulación europea de las plataformas de intermediarios digitales en la era de la economía colaborativa. Revista Crítica de Derecho Inmobiliario 94 (765), 275-330. 